\title{
Methods for obtaining and processing biocompatible medical materials
}

\author{
Mikhail Ivanov ${ }^{1}$, Elena Poddubskaya ${ }^{2}$, and Nadezda Uchevatkina ${ }^{2, *}$ \\ ${ }^{1}$ Federal State Budget Institution of Higher Education «Financial University under the Government of \\ the Russian Federation», Leningradsky Prospekt 17 Moscow, 125993, Russian Federation \\ ${ }^{2}$ Federal State Budgetary Institution «National Medical Research Center for Preventive Medicine» of \\ the Ministry of Healthcare of the Russian Federation, Petroverigskiy lane 10 str 3Moscow, 101990, \\ Russian Federation
}

\begin{abstract}
The article is devoted to the scientific problem associated with the production and processing of biocompatible medical materials with improved mechanical and functional properties. Processing biocompatible materials is one of the key processes to achieve operational properties. The study presents the results of microhardness and microstructure, which showed that electroplastic treatment, together with subsequent annealing, helps to obtain a homogeneous nanocrystalline structure and improve a number of operational properties that play an important role when using biocompatible medical materials.
\end{abstract}

Scientific and technological progress and human production activities are attracted to the increased requirements for materials. One of them is minimization of the size of objects, while maintaining the complex of physical and mechanical properties of materials. The use of thermomechanical processing methods can allow this to be implemented in industry.

Nanostructured alloys are promising materials for various applications. The formation of the shape and size of products, as well as the level of physical and mechanical properties. These materials must simultaneously have high strength and resistance to wear and biocompatibility. This should include both bulk and surface hardening. All the above characteristics make it possible to achieve, using, accordingly, the electroplastic effect (EPE), pulsed laser irradiation and ion implantation.

EPE was detected at real single current pulses with a density of $\sim 105 \mathrm{~A} / \mathrm{cm} 2$ and a duration of 10-4 sec during the deformation of zinc and large-crystalline titanium single crystals by tension and compression. He manifested himself in spasmodic elongated samples. However, the manifestation of EPE in intermetallic devices with low electrical conductivity, such as TiNi, has not yet been known. Moreover, the role of the nanostructure in such an alloy has never been studied before.

EPE is less universal than the joule effect, because in contrast, it exists only during plastic deformation of the material. However, it is energetically less capacious, because

\footnotetext{
*Corresponding author: uchevatkina@yandex.ru
} 
current is introduced only into the metal deformation zone. The advantages of electroplastic technology for metal processing include the following: the possibility of influencing the material, as well as the creation of new technologies that allow obtaining materials with changing properties, high speed and effectiveness of the impact, the possibility of combined exposure using the joule effect. associated temperatures.

To create extreme loads on the surface of the material, the laser shock wave method is used, in which the laser radiation energy is rapidly transformed into various types of mechanical energy, mainly in the form of shock wave energy. The dislocation-diffusion mechanism that occurs when exposed to a laser shock wave begins to act when the defect is in a field of sufficiently high external stresses, since the emission of dislocation loops is a threshold effect, in contrast to the process of emission of vacancies. A characteristic method concomitant with dislocation healing is the dispersion of the matrix in the region around the pores, which determines the transition from the dislocation mechanism to isotropic viscous healing of pores and cracks. Another positive factor of laser processing is the possibility of obtaining a gradient structure. The paper presents the results of studies of microhardness, corrosion resistance, fatigue strength.

In the last decade, alloys exhibiting the shape memory effect have been widely used. Alloys with memory form in various fields of technology. The device for the accumulation of world practice [1-10] is a medical technique. SPFs based on titanium nickelide TiNi possess the highest complex of functional properties. The use of titanium nickelide in medicine, in particular, is due to the unique combination of functional properties and the shape of the memory with high corrosion resistance in liquid media of the human body, as well as with the features of its superelastic mechanical behavior, similar to the mechanical effect of bone tissue. It is fully biocompatible.

Functional properties of shape memory alloys are structurally sensitive. Therefore, thermomechanical processing is an effective way to control them. Using thermomechanical processing, which creates various types of microstructures, the properties of alloys with shape memory are regulated within wide limits, and at the same time, a set of properties that cannot be achieved by conventional heat treatment methods can be obtained. The reserve for controlling the functional properties of shape memory alloys using thermomechanical processing can be expanded if we use the possibility of forming a nanocrystalline and amorphous structure in $\mathrm{Ti}-\mathrm{Ni}$ alloys by electroerosion methods.

The mechanism of electroplastic processing in materials with structural-phase transformations consists in the interaction of conduction electrons with structural elements that have excess stored energy (accumulations of dislocations, impurity atoms, vacancies). The control of this process can lead to increased hardness (due to dispersion hardening), viscosity (due to the production of fine grains), strength, ductility, and wear resistance.

The use of electric heating allows you to radically increase productivity, mechanize and automate the heat treatment process, embed thermal installations directly in the line for machining parts.

To obtain biocompatible nanostructured materials, the method of intensive plastic deformation (IPD) is often used, which allows one to obtain bulk non-porous nanocrystalline metals and alloys. Using this method, nano- and subcrystalline structures are formed in aluminum, iron, magnesium, tungsten, nickel, titanium and their alloys. Such a structure leads to a change in physical and mechanical properties (a significant increase in strength while maintaining ductility, improved wear resistance, the manifestation of highspeed and low-temperature superplasticity). The process consists in deformation with large degrees of deformation at relatively low temperatures (below 0.3-04, Tm) under conditions of high positive pressures. The method is based on the principle of the formation of a highly fragmented and disoriented structure due to large deformations. During IPD, amorphization of the material is also possible. 
Studies of domestic and foreign scientists on various materials have shown that the use of electrical discharge machining, drawing of various materials leads to an increase in their ductility and reduction of deformation forces, as well as to improvement of mechanical properties, while reducing energy costs while increasing the resource of deforming equipment.

There are three main methods of supplying current during one of the types of electroerosive processing of electroplastic deformation: with supplying current from roll to roll, with supplying current with one pole through a sliding contact to the workpiece deformation zone between rolls, and with a second pole through rolls and with two sliding current supplying contacts before and after the zone of electroplastic deformation between the rolls. The first method, despite the observance of the conditions for concomitant deformation by rolling with an electroplastic action by current, is not quite effective from the point of view of the complete study of the material for thick workpieces by current. In the second method, there are no restrictions on the thickness of the sample, but the streamlines do not coincide with the direction of movement of the zones of plastic flow of material between the rollers. In addition, the rolls remain under current, which, in addition to the technical difficulties associated with the need to isolate the rolls from the mill, is also associated with electrical discharge processes on the rolls. The implementation of the third scheme is associated with an incomplete value of EPE (electroplastic effect), but this drawback is compensated by the ease of supplying current and the safety of the mill work rolls, which is important to guarantee long-term operation of the mill for electroerosive processing.

The nitinol alloy of composition $\mathrm{Ti}_{49.3} \mathrm{Ni}_{50.7}$, a sample after EPD with a certain degree of deformation and size, was chosen as the object of research considered in this article. The degree of deformation is e $=1.5-1.8$, the dimensions of the samples are $50 \times 10 \times 0.41$.

Rolling was carried out on a rolling mill for electroplastic rolling and rolling of wire, equipped with a pulse current generator. The diameter of the steel rolls is $120 \mathrm{~mm}$, the length is $200 \mathrm{~mm}$. Rolling was carried out at room temperature with a speed of $3.5 \mathrm{~cm} / \mathrm{s}$ in a step-by-step mode with controlled single-shot reduction in thickness of no more than 25 microns. To supply and remove current, a sliding contact was used (negative pole to the deformation zone and one of the rolls (positive pole). For EPD, the method of electroplastic rolling in crossed electromagnetic fields was applied when applying current to the workpiece and to the rolls. To obtain current pulses, special pulse generators, in which the inductive and capacitive storage devices serve as an energy-consuming element. When choosing the size and shape of the sample, it was not only the possibility of conducting the electric erosion treatment, but the need for further research - tension, microhardness measurements, microstructural analysis, taking into account the economy of scarce and expensive alloys. Another criterion for choosing a sample was the power of the equipment, namely the current density. For samples with a cross-sectional area of 5 and $50 \mathrm{~mm}$, different power is required from a current generator for performing electrical discharge machining. For a sample with a cross-sectional area of $50 \mathrm{~mm}$, the current density should be higher, but this will lead to a lack of power equipment for the existence of electric pulse processing.

As a result, rectangular squared samples $0.5 \times 50 \times 10 \mathrm{~mm}^{3}$ in size were selected. The maximum exposure duration and current density were chosen for logical reasons, so that the sample would not oxidize and remain solid, but at the same time get the result with maximum microhardness. The intermediate values of the parameters of electric discharge machining were chosen experimentally in such a way that the processing time was minimal and the current density did not exceed the maximum value on the generator. Two main modes were investigated: at a selected current density of $110 \mathrm{~A} / \mathrm{mm}^{2}$, the time was varied, 
and at the optimum shutter speed, the current density was varied. The measured characteristic, which was used to judge the properties of the alloy, was microhardness.

Electroerosive treatment includes the following modes: constant current density mode with changing time and constant time mode with changing current density. The optimal mode was selected by measuring the microhardness of the sample (Figure 1) after electroerosion treatment. The exposure time for the samples was from 1 to $10 \mathrm{~s}$ at a current density of 90-110 A / $\mathrm{mm}^{2}$, pulse duration $100 \mu \mathrm{s}$, frequency $1000 \mathrm{~Hz}$. The influence of accumulated time was also carried out, i.e. some more time was passed through the sample. The current density was varied from $100 \mathrm{~A} / \mathrm{mm}^{2}$ to $267 \mathrm{~A} / \mathrm{mm}^{2}$ with an exposure time of 3, 4 and $5 \mathrm{sec}$, a pulse duration of $110 \mu \mathrm{s}$, and a frequency of $910 \mathrm{~Hz}$. During the experiments, the behavior of the material was manifested, the temperature front begins in the center and moves to the clamps. A deflection of the sample appears at a density of more than $150 \mathrm{~A} / \mathrm{mm}^{2}$.

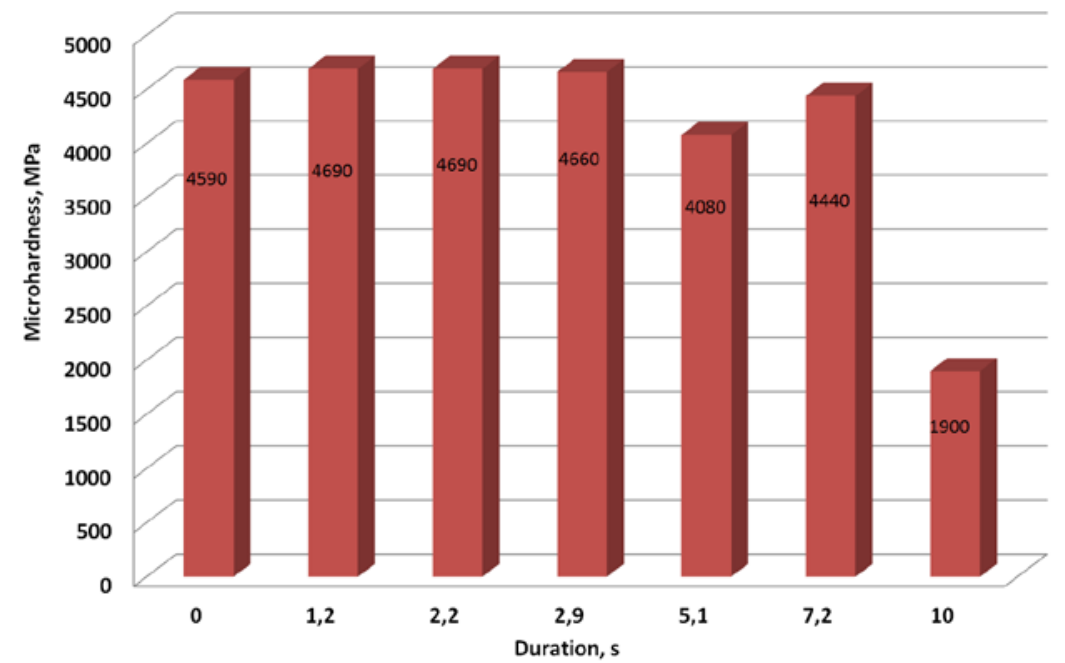

Fig. 1. Microhardness of a deformed alloy after electric discharge machining $\left(\mathrm{j}=100 \mathrm{~A} / \mathrm{mm}^{2}, \tau=\right.$ $100 \mu \mathrm{s}, \nu=910 \mathrm{~Hz})$

Before EDM, the microhardness of the alloy was 4590. With an increase in the processing time to $3,2 \mathrm{sec}$, the microhardness remains almost unchanged, and at 5,1 sec there is a slight drop in microhardness to $4080 \mathrm{MPa}$, then 7,2 sec we see a decrease in microhardness relative to the original at $10 \mathrm{sec}$ we see a sharp drop in microhardness. After analyzing the results, we can say that the interval from $3 \mathrm{~s}$ to $5 \mathrm{sec}$ is most interesting, since there is a drop-in microhardness, which may indicate that the treatment stimulates an increase in technological plasticity and a decrease in strength characteristics.

Studies of the microstructure after EDM are shown in Figure 2, which shows the microstructure in dark fields (a) after EDM and (b) after annealing at $450{ }^{\circ} \mathrm{C}$ and holding time of $60 \mathrm{~min}$ and microdiffraction of the TiNi alloy after electroplastic deformation with a degree of deformation of $\mathrm{e}=1.8$ and $\mathrm{ESR}$ at $\mathrm{t}=3 \mathrm{~s}, \mathrm{i}=110 \tau=100 \mu \mathrm{s}, \mathrm{v}=910 \mathrm{~Hz}$.

Studies have shown that a homogeneous nanocrystalline structure is formed in the alloy after electric discharge machining (Fig. 2, a).

The nanocrystalline structure (as a result of the dispersion of the ultrafine-grained structure) is represented by nanoscale spotted contrast in a bright field and point diffraction rings (or their parts) in the diffraction pattern.

Electroerosive treatment of a deformed alloy at $\mathrm{t}=3 \mathrm{~s}, \mathrm{i}=110 \tau=100 \mu \mathrm{s}, v=910 \mathrm{~Hz}$ leads to nanocrystallization of amorphous regions and growth of crystallites to an average 
size of $35 \mathrm{~nm}$, determined from the dark-field image of the structure in the reflection of the high-temperature phase B2 (Fig. 2).

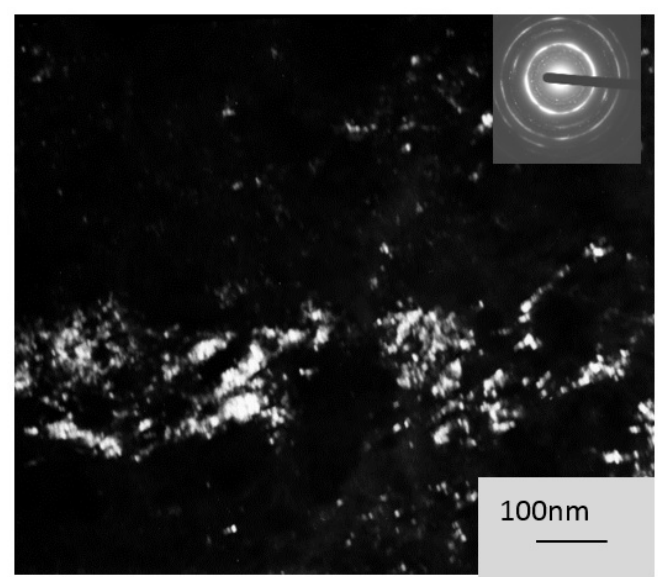

a)

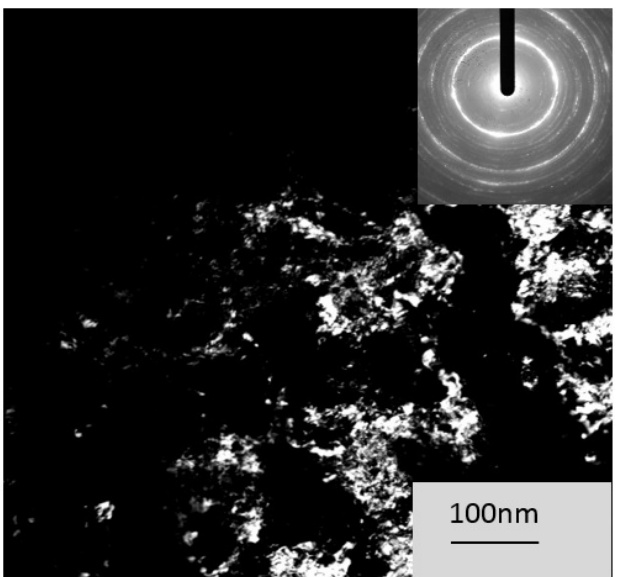

б)

Fig. 2. Microstructure and microdiffraction of a TiNi alloy subjected to a - electrical discharge machining, and $\mathrm{b}$ - electrical discharge machining and subsequent annealing at $450{ }^{\circ} \mathrm{C}$.

\section{Conclusion}

In general, we can conclude that when comparing the two structures (fig 2, a) and (fig 2, b), we obtained the structure and properties of the alloy no worse than after annealing, therefore, the use of the electroplastic processing method contributes to the production of a homogeneous nanocrystalline structure. Preliminary experiments also showed a weak sensitivity of the electroplastic effect to the frequency and duty cycle of the pulse current, and a strong dependence on the density and duration of the pulse. A twofold increase in current density (from 80 to $168 \mathrm{~A} / \mathrm{mm}^{2}$ ) can lead to a more than twofold increase in deformation. An increase in the duration of the current pulse has a smaller effect on deformability, and the pulse duration from 80 to $160 \mu$ s at current densities of 80 and $120 \mathrm{~A} / \mathrm{mm}^{2}$ does not increase the deformability of the alloy, but at a current density of $168 \mathrm{~A} / \mathrm{mm}^{2}$ it significantly increases it. Analysis of experimental data shows that in all cases, rolling with current is more efficient $(e=1,65-1,81)$ than without current $(0,51-0,53)$. Maximum deformation with current is greater than deformation without current, more than 3 times. An increase in current density and pulse duration also stimulates an increase in technological plasticity.

\section{Reference}

1. Kaynak Y, Karaca H. E., Noebe R. D., Jawahir I.S. Tool-wear analysis in cryogenic machining of NiTi shape memory alloys: a comparison of tool-wear performance with dry and MQL machining. Wear 306:51-63 (2013).

2. Weinert K, Petzoldt V. Machining NiTi micro-parts by micro-milling. Mater Sci Eng A 481-482:672-675 (2008).

3. Piquard R, D'Acunto A, Laheurte P, Dudzinski D. Micro-end milling of NiTi biomedical alloys, burr formation and phase transformation. Precis Eng 38:356-364 (2014). 
4. Kong M.C., Axinte D, Voice W. Challenges in using waterjet machining of NiTi shape memory alloys: an analysis of controlled-depth milling. J Mater Process Technol 211:959-971 (2011).

5. Frotscher M, Kahleyss F, Simon T, Biermann D, Eggeler G. Achieving small structures in thin NiTi sheets for medical applications with water jet and micro machining: a comparison. J Mater Eng Perform 20:776-782 (2011).

6. Frotscher M, Kahleyss F, Simon T, Biermann D, Eggeler G. Achieving small structures in thin NiTi sheets for medical applications with water jet and micro machining: a comparison. J Mater Eng Perform 20:776-782 (2011).

7. Fu C, Liu J, Guo A. Statistical characteristics of surface integrity by fiber laser cutting of Nitinol vascular stents. Appl Surf Sci 353:291-299 (2015).

8. Hung C-H, Chang F-Y, Chang T-L, Chang Y-T, Huang $K-W$, Liang P-C. Micromachining NiTi tubes for use in medical devices by using a femtosecond laser. Opt Lasers Eng 66:34-40 (2015).

9. $\mathrm{Ng}$ C, Chan C, Man H, Waugh D, Lawrence J. NiTi shape memory alloy with enhanced wear performance by laser selective area nitriding for orthopaedic applications. Surf Coat Technol 309:1015-1022 (2017).

10. Liu J-X, Yang D-Z, Shi F, Cai Y-J. Sol-gel deposited TiO2 film on NiTi surgical alloy for biocompatibility improvement. Thin Solid Films 429:225-230 (2003). 